\title{
Thermodynamic properties of nuclear matter with three-body forces
}

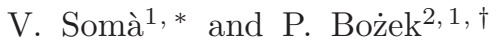 \\ 1 Institute of Nuclear Physics PAN, PL-31-342 Kraków, Poland \\ ${ }^{2}$ Institute of Physics, Rzeszów University, PL-35-959 Rzeszów, Poland
}

(Dated: November 3, 2018)

\begin{abstract}
We calculate thermodynamic quantities in symmetric nuclear matter within the self-consistent Green's functions method including three-body forces. The thermodynamic potential is computed directly from a diagrammatic expansion, implemented with the CD-Bonn and Nijmegen nucleonnucleon potentials and the Urbana three-body forces. We present results for entropy and pressure up to temperatures of $20 \mathrm{MeV}$ and densities of $0.32 \mathrm{fm}^{-3}$. While the pressure is sensitive to the inclusion of three-body forces, the entropy is not. The unstable spinodal region is identified and the critical temperature associated to the liquid-gas phase transition is determined. When three-body forces are added we find a strong reduction of the critical temperature, obtaining $T_{c} \simeq 12 \mathrm{MeV}$.
\end{abstract}

PACS numbers: 21.30.Fe, 21.65.Mn

The determination of thermodynamic properties of hot nuclear matter is extremely relevant for its applications in astrophysics and heavy-ion reactions. Pressure and entropy at finite temperature are crucial ingredients in the modeling of core-collapse supernovae and protoneutron stars, which involve processes characterized by densities up to several time the nuclear saturation density and temperatures up to few tens of MeV's. The nuclear equation of state (EOS) plays an important role also in the interpretation of nucleus-nucleus collisions, in which a hot and dense state of matter is formed. Of particular interest is the study of a possible phase transition occurring at subsaturation densities. Because of the van der Waals nature of nucleon-nucleon (NN) interactions, at low densities and high temperatures nuclear matter is expected to undergo a first-order transition to a gas phase [1]. It is claimed that an evidence for such behavior is found in intermediate energy heavy ion collisions, as a plateau of the caloric curve derived for the light fragments as a function of the reaction energy [2].

The liquid-gas transition in nuclear matter presents some differences with respect to the case of finite nuclei where, as the temperature increases, Coulomb forces together with the decrease of the surface tension trigger the onset of mechanical instabilities. One can try to relate the limiting temperature of nuclei $T_{l}$ to the critical temperature in infinite matter $T_{c}$ by properly taking into account Coulomb and surface effects [3]. Different estimates from effective models based on Skyrme forces [4] and more microscopic approaches [5, 6] are in agreement and yield a ratio $T_{l} / T_{c}$ of about $1 / 3$.

A reliable many-body theory is called for the interpretation of the heavy ion reactions in the goal of extracting the equation of state and the critical point of the nuclear liquid-gas phase transition [7] and in order to extrapolate to higher densities and arbitrary isospin asymme-

\footnotetext{
*Electronic address : vittorio.soma@ifj.edu.pl
}

${ }^{\dagger}$ Electronic address : piotr.bozek@ifj.edu.pl tries. There exist only few realistic calculations of the nuclear matter EOS at finite temperature. The variational approach, which yields reliable results at $T=0$, is usually extended to finite temperatures by neglecting the modifications of the correlation functions [8]. There are attempts of developing a finite $T$ variational technique [9] but no calculations of thermodynamic quantities are available so far. The Brückner-Hartree-Fock method generalized by means of the Bloch-de Dominicis formalism has been applied to finite temperature nuclear matter 10, 11]. The Green's functions in-medium T-matrix [12, 13] approach is suitable for computing consistently microscopic properties and thermodynamic observables and naturally takes into account finite temperature correlations [14]. Thermodynamic relations such as the Hugenhotlz-van Hove and Luttinger identities [15, 16] are automatically fulfilled by the $\Phi$-derivable T-matrix approximation [17, 18].

In two recent papers we presented the first calculations of entropy and pressure at finite temperature within the thermodynamically consistent T-matrix approach 19] and the first results which include three-body forces in the finite temperature Green's functions method in nuclear matter [20]. Following these works we compute here pressure and entropy without and with three-body forces in the case of symmetric nuclear matter for two different realistic NN potentials, in particular addressing the unstable region related to the liquid-gas phase transition.

Let us briefly recall the adopted computational method, whose details can be found in Refs. [19, 20]. We solve the set of coupled equations which involve the calculation of the T-matrix, summing the ladder diagrams at all orders, the determination of the nucleon self-energy and the single-particle Green's function. The full off-shell propagation of particles in the medium is taken into account. We employ two different realistic nucleon-nucleon interactions, the CD-Bonn [21] and the Nijmegen 22] parameterizations, together with the semi-microscopic Ur- 
bana three-nucleon potential [23]. Three-body forces, necessary for a reliable description of the saturation properties in symmetric nuclear matter, are included via an effective two-body interaction derived after averaging out the third particle. The effect on the two remaining nucleons results in a mean field whose two parameters are fixed by requiring the correct saturation density and binding energy. The range of temperatures and densities studied is well above the superfluid transition in symmetric nuclear matter [24].

After calculating the single-particle propagator $G$ and the nucleon self-energy $\Sigma$ from the iterative scheme of the T-matrix equations one can compute the grand canonical potential, expressed as

$$
\Omega=-\operatorname{Tr}\left\{\ln \left[G^{-1}\right]\right\}-\operatorname{Tr}\{\Sigma G\}+\Phi .
$$

The generating functional $\Phi$ has a diagrammatic expansion similar to the one for the interaction energy in the T-matrix approximation, differing by a factor $1 / n$ ( $n$ is the number of interaction lines) in front of each diagram (see Fig. 1). Therefore we derive $\Phi$ from the expression

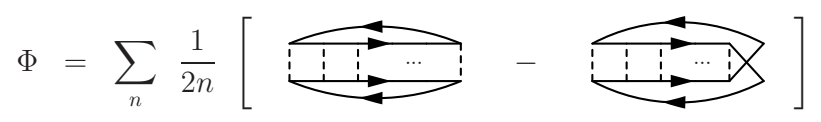

FIG. 1: Diagrammatic expansion of the generating functional $\Phi$. The sum includes ladder-type diagrams up to infinite order ( $n$ is the number of interaction lines in each diagram).

for $\left\langle H_{\text {int }}\right\rangle$ by multiplying the potential $V$ by a numerical factor $\lambda$ and integrating

$$
\Phi=\int_{0}^{1} \frac{d \lambda}{\lambda}\left\langle H_{\text {int }}\left(\lambda V, G_{\lambda=1}\right)\right\rangle,
$$

where $G$ is the dressed single-particle propagator computed in the system with the full strength interaction. The expectation value $\left\langle H_{\text {int }}\right\rangle$ itself can be easily obtained from the $T$ matrix [19]. From the grand canonical potential (dividing by the volume $\mathcal{V}$ ) we obtain the pressure

$$
P=-\frac{\Omega}{\mathcal{V}}
$$

which is then computed directly from its diagrammatic expansion without the use of numerical derivatives.

In Figs. 2 and 3 we present the pressure for symmetric nuclear matter without and with three-body forces for the CD-Bonn and for the Nijmegen potential respectively. Results are shown for different temperatures as function of the density. When looking at the curves obtained with the two-body NN potentials (upper panels) we notice that they cross the thermodynamically forbidden region in which the derivative of the pressure with respect to the density is negative. For both CD-Bonn and
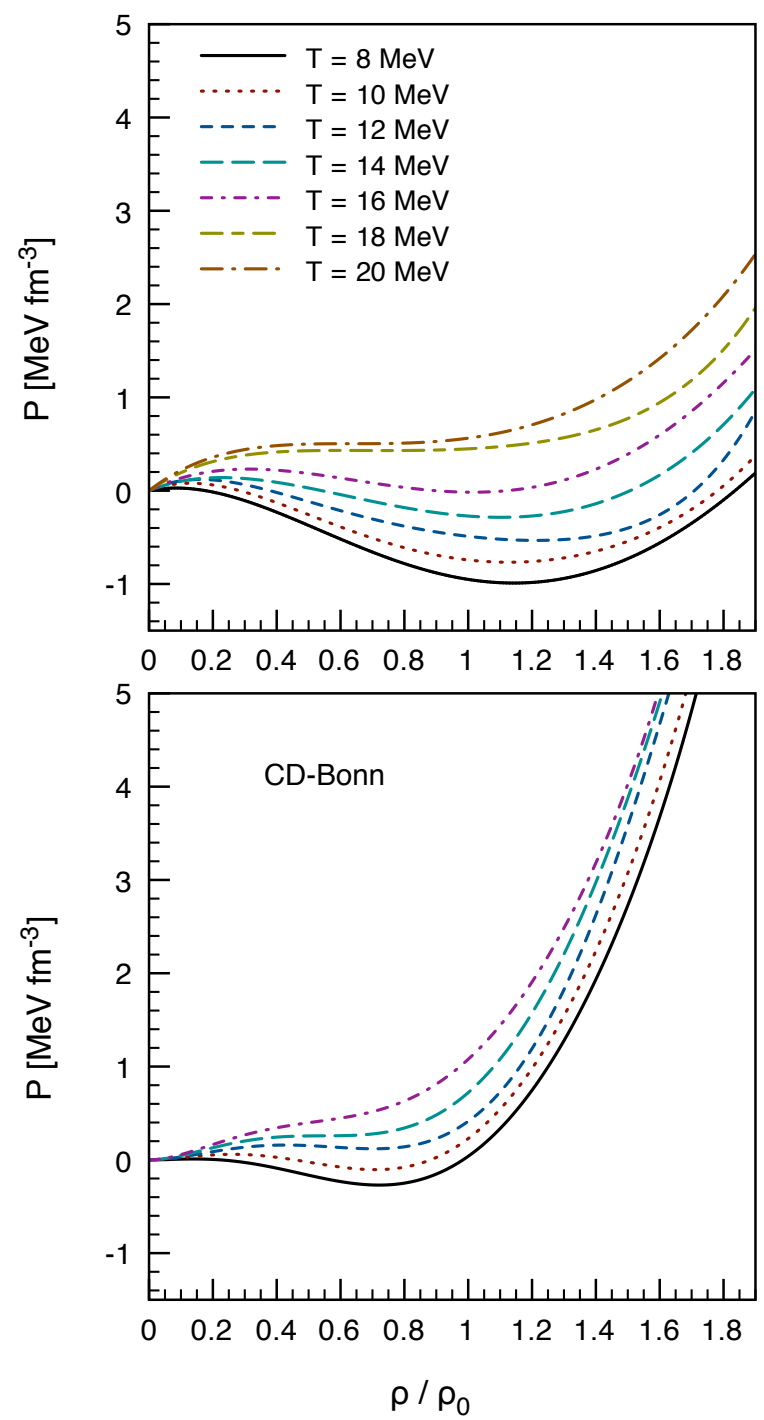

FIG. 2: (Color online) Pressure as function of density in symmetric nuclear matter for different temperatures with the CDBonn potential (upper panel) and the CD-Bonn potential plus three-body forces (lower panel).

Nijmegen interactions the inclusion of three-body forces (lower panels) leads to a stiffening of the dependence of the pressure on density. The spinodal instability region below saturation density is still present but gets substantially reduced.

In order to address more in details the critical behavior and the effect of three-body forces on the critical temperature for the liquid-gas phase transition we study the limits of the instability region in the density-temperature plane. The spinodal region is characterized by the negative derivatives of the pressure and the chemical potential

$$
\left.\frac{\partial P}{\partial \rho}\right|_{T}<0,\left.\quad \frac{\partial \mu}{\partial \rho}\right|_{T}<0 .
$$



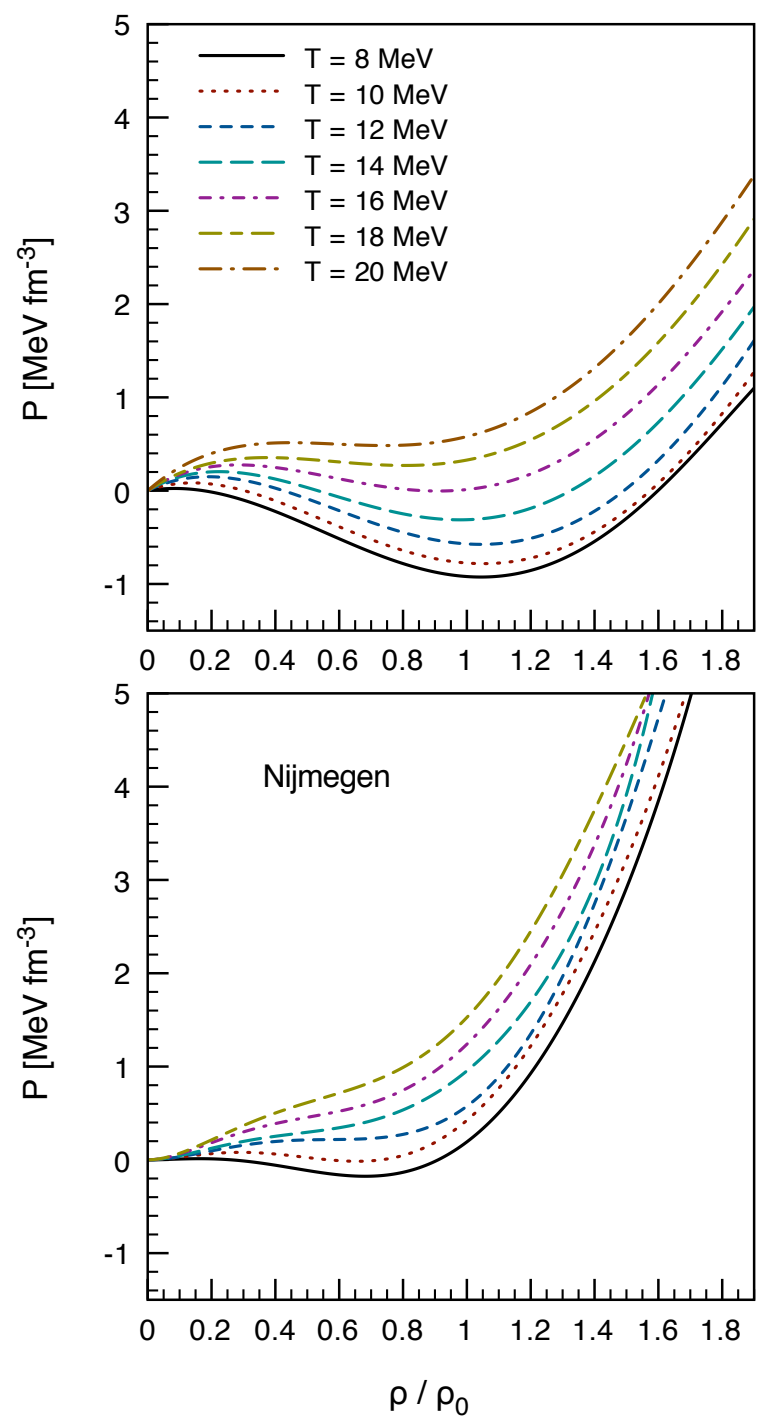

FIG. 3: (Color online) Pressure as function of density in symmetric nuclear matter for different temperatures with the Nijmegen potential (upper panel) and the Nijmegen potential plus three-body forces (lower panel).

Inside this region the system is unstable and tends to separate in two different phases, gas at a lower and liquid at a higher density. The two phases may coexist in an interval of densities and temperatures up to the point in which

$$
P\left(\rho_{\text {gas }}\right)=P\left(\rho_{\text {liquid }}\right)
$$

and

$$
\mu\left(\rho_{\text {gas }}\right)=\mu\left(\rho_{\text {liquid }}\right) .
$$

The two regions end up in a coincident point which defines the critical density and temperature of the liquidgas phase transition.

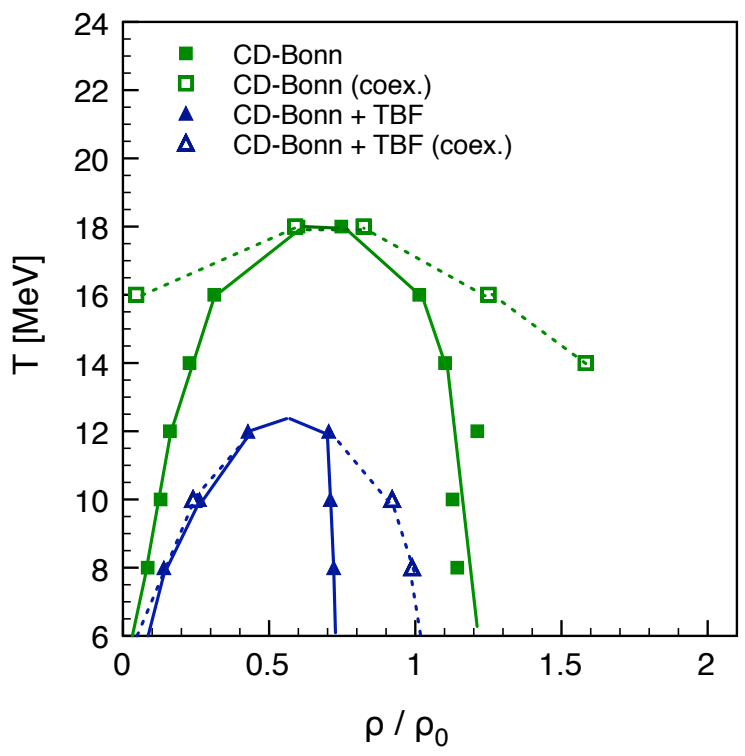

FIG. 4: (Color online) Coexistence and spinodal lines for the CD-Bonn potential without and with three-body forces.

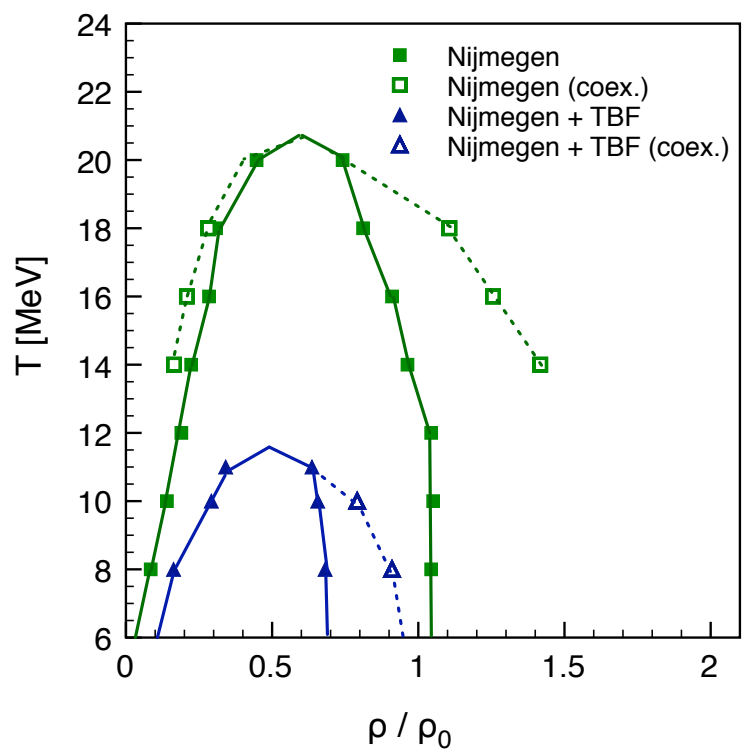

FIG. 5: (Color online) Coexistence and spinodal lines for the Nijmegen potential without and with three-body forces.

We compute the spinodal and the coexistence lines for the CD-Bonn and the Nijmegen potential without and with the inclusion of three-body forces. The limits of the spinodal region defined by Eq. (4) are found as local maxima and minima of the pressure. The conditions on the pressure and chemical potential are equivalent for a one-component system such as symmetric or pure neutron matter. For the case of arbitrary isospin asymme- 
tries one should in principle consider both of them, but there is evidence that chemical and mechanical instabilities coincide also for asymmetric nuclear matter 25].

We use two methods for determining the spinodal line: we compute the pressure derivative directly from the interpolation of $P$ and apply the one suggested by Baldo and Ferreira [10] which consists in plotting the chemical potential as a function of the pressure, looking for the back bending of the curves. The results obtained with the two techniques do coincide, confirming the stability of the calculations even in the low density regime. The critical lines are displayed in Figs. 4 and 5 for CD-Bonn and Nijmegen respectively. At low temperatures the unstable and coexistence phases are present over a large range of densities. As the temperature increases the instability region becomes smaller ending up with the point defining the critical temperature for the liquid-gas transition. The critical temperature is $T_{c}=18 \mathrm{MeV}$ for the CD-Bonn and $T_{c}=20.5 \mathrm{MeV}$ for the Nijmegen potential. Both the spinodal and the coexistence regions get strongly reduced when three-body forces are included, with the critical point at $T_{c}=12.5 \mathrm{MeV}$ when CD-Bonn is employed and $T_{c}=11.5 \mathrm{MeV}$ if we consider the Nijmegen potential.

Another quantity which characterizes the liquid-gas phase transition is a dimensionless parameter that comprises the critical pressure, density and temperature, $P_{c} /\left(\rho_{c} T_{c}\right)$, which assumes the value $3 / 8$ for a van der Waals equation of state. In Table 1 we summarize these

\begin{tabular}{|c||c|c|c|c|}
\hline potential & $T_{c}(\mathrm{MeV})$ & $\rho_{c}\left(\mathrm{fm}^{-3}\right)$ & $P_{c}\left(\mathrm{MeV} \mathrm{fm}^{-3}\right)$ & $\frac{P_{c}}{\rho_{c} T_{c}}$ \\
\hline \hline CD-Bonn & 18 & 0.107 & 0.43 & 0.22 \\
\hline CD-Bonn + TBF & 12.5 & 0.096 & 0.14 & 0.12 \\
\hline Nijmegen & 20.5 & 0.094 & 0.50 & 0.26 \\
\hline Nijmegen + TBF & 11.5 & 0.088 & 0.15 & 0.14 \\
\hline
\end{tabular}

TABLE I: Critical values of the temperature, density and pressure of the liquid-gas phase transition for the CD-Bonn and Nijmegen potentials without and with three-body forces.

critical quantities for the various potentials without and with three-body forces. The values for the CD-Bonn and the Nijmegen potentials are rather similar, with a strong decrease of the dimensionless parameter after the inclusion of three-body forces, signaling a departure from the van der Waals equation of state.

Our estimates with the two-body nucleon-nucleon interactions only are in agreement with other microscopic calculations. Rios et al. 26] use a self consistent Green's functions approach but compute the free energy from the Carneiro-Pethick quasiparticle entropy [27], obtaining for the CD-Bonn potential a critical temperature $T_{c}=18.5 \mathrm{MeV}$ and $P_{c} /\left(\rho_{c} T_{c}\right)=0.20$. When using the Argonne V18 parameterization, however, they find that $T_{c}$ is reduced to about $11 \mathrm{MeV}$ and $P_{c} /\left(\rho_{c} T_{c}\right)=0.14$. Baldo and Ferreira [10] performed calculations within the Bloch-de Dominicis finite temperature generalization of the Brückner-Hartree-Fock method. Using the Argonne V18 potential they estimate the critical temperature to be $T_{c} \simeq 21 \mathrm{MeV}$. However, when they include the Urbana three-nucleon potential in the calculations they find, in contrast with our result, that three-body forces do not strongly affect the critical temperature, which is reduced to $T_{c} \simeq 20 \mathrm{MeV}$. Other calculations within the Bloch-de Dominicis formalism [28] or the relativistic Dirac-Brückner-Hartree-Fock approach [5, 29], on the other hand, yield lower values of $T_{c}$, respectively 9,12 and $10.4 \mathrm{MeV}$, closer to our result with three-body forces.

We compute the entropy per particle in the interacting system from the thermodynamic relation

$$
\frac{S}{N}=\frac{1}{T}\left[\frac{E}{N}+\frac{P}{\rho}-\mu\right] .
$$

The results for symmetric nuclear matter at $\rho=\rho_{0}$ are shown in Fig. 6 for different temperatures. The

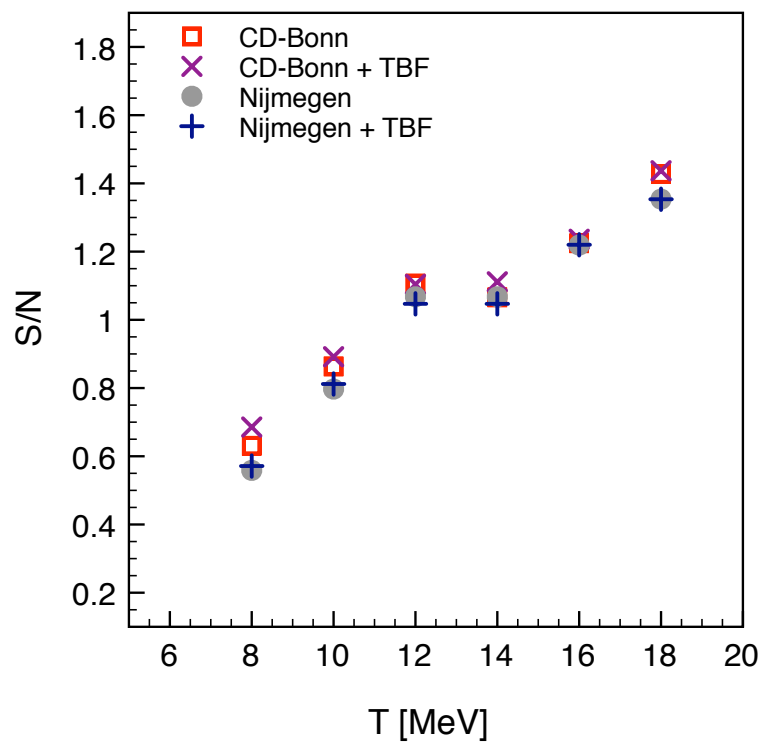

FIG. 6: (Color online) Entropy per particle for symmetric nuclear matter at $\rho=\rho_{0}$ with the CD-Bonn and Nijmegen potentials without and with three-body forces.

effects of three-body forces are very small, as well as the dependence on the nucleon-nucleon potential. The entropy appears to be independent of the details of the interaction, supporting the conclusion that it is not much affected by nucleon correlations. The reliability of calculations of the entropy at the level of a quasiparticle approximation has been checked also by Rios et al. [30] using the Carneiro-Pethick dynamical quasiparticle formula [27]. 
Existing estimates of the critical temperature for the liquid-gas transition in infinite nuclear matter are in the range $T_{c} \simeq 9-21 \mathrm{MeV}$. Within the finite temperature Green's functions approach we calculate thermodynamic properties of symmetric matter and study the spinodal instability region related to the first-order phase transition. We observe that three-body forces have a strong effect on the pressure of the interacting system, reducing the size of the unstable region in the density-temperature plane. With the CD-Bonn and the Nijmegen potential we find that the critical temperature decreases respectively from $T_{c}=18 \mathrm{MeV}$ to $T_{c}=12.5 \mathrm{MeV}$ and from $T_{c}=20.5 \mathrm{MeV}$ to $T_{c}=11.5 \mathrm{MeV}$. In all cases the critical density lies in the range $\rho_{c} \simeq 0.09-0.11 \mathrm{fm}^{-3}$. The pressure at which the phase transition takes place decreases from $P_{c} \simeq 0.43-0.50 \mathrm{MeV} \mathrm{fm}^{-3}$ to about $P_{c}=0.15 \mathrm{MeV} \mathrm{fm}^{-3}$ after the inclusion of the three- nucleon potential.

Three-body forces do not affect the entropy, which turns out to be independent of the potential used in the calculations. This last results confirms that entropy is not much sensitive to nucleon-nucleon correlations (cf. Refs. [19, 30]).

\section{Acknowledgements}

Research supported in part by the Polish Ministry of Science and Higher Education, grant N N202 1022 33. V. S. acknowledges the Academic Computer Center CYFRONET of the AGH University of Science and Technology in Kraków for benefiting from the use of its high performance computers.
[1] G. Bertsch and P. J. Siemens, Phys. Lett. B126, 9 (1983).

[2] J. B. Natowitz, R. Wada, K. Hagel, T. Keutgen, M. Murray, A. Makeev, L. Qin, P. Smith, and C. Hamilton, Phys. Rev. C 65, 034618 (2002).

[3] S. Levit and P. Bonche, Nucl. Phys. A437, 426 (1985).

[4] J. B. Natowitz, K. Hagel, Y. Ma, M. Murray, L. Qin, R. Wada, and J. Wang, Phys. Rev. Lett. 89, 212701 (2002).

[5] B. Ter Haar and R. Malfliet, Phys. Rev. Lett. 56, 1237 (1986).

[6] M. Baldo, L. S. Ferreira, and O. E. Nicotra, Phys. Rev. C 69, 034321 (2004), nucl-th/0402006.

[7] P. Chomaz, M. Colonna, and J. Randrup, Phys. Rept. 389, 263 (2004).

[8] B. Friedman and V. R. Pandharipande, Nucl. Phys. A361, 502 (1981).

[9] A. Mukherjee and V. R. Pandharipande, Phys. Rev. C 75, 035802 (2007), nucl-th/0609058.

[10] M. Baldo and L. S. Ferreira, Phys. Rev. C 59, 682 (1999).

[11] W. Zuo, Z. H. Li, U. Lombardo, G. C. Lu, and H. J. Schulze, Phys. Rev. C 73, 035208 (2006).

[12] W. H. Dickhoff, Phys. Rev. C 58, 2807 (1998).

[13] P. Bożek, Phys. Rev. C 59, 2619 (1999), nucl-th/9811073.

[14] L. P. Kadanoff and G. Baym, Quantum Statistical Mechanics (Bejamin, New York, 1962).

[15] N. Hugenholtz and L. Von Hove, Physica 24, 363 (1958).

[16] J. M. Luttinger, Phys. Rev. 119, 1153 (1960).

[17] G. Baym, Phys. Rev. 127, 1391 (1962).
[18] P. Bożek and P. Czerski, Eur. Phys. J. A11, 271 (2001), nucl-th/0102020.

[19] V. Somà and P. Bożek, Phys. Rev. C 74, 045809 (2006), nucl-th/0604030.

[20] V. Somà and P. Bożek, Phys. Rev. C 78, 054003 (2008), 0808.2929.

[21] R. Machleidt, Phys. Rev. C 63, 024001 (2001), nuclth/0006014.

[22] V. G. J. Stoks, R. A. M. Klomp, C. P. F. Terheggen, and J. J. de Swart, Phys. Rev. C 49, 2950 (1994), nuclth/9406039.

[23] J. Carlson, V. Pandharipande, and R. Wiringa, Nucl. Phys. A401, 59 (1983).

[24] P. Bożek, Phys. Lett. B551, 93 (2003), nucl-th/0202045.

[25] J. Margueron and P. Chomaz, Phys. Rev. C 67, 041602 (2003), nucl-th/0212082.

[26] A. Rios, A. Polls, A. Ramos, and H. Muther, Phys. Rev. C 78, 044314 (2008), 0805.2318.

[27] G. M. Carneiro and C. J. Pethick, Phys. Rev. B 11, 1106 (1975).

[28] C. Das, R. K. Tripathi, and R. Sahu, Phys. Rev. C 45, 2217 (1992).

[29] H. Huber, F. Weber, and M. K. Weigel, Phys. Rev. C 57, 3484 (1998).

[30] A. Rios, A. Polls, A. Ramos, and H. Muther, Phys. Rev. C 74, 054317 (2006), nucl-th/0605080. 